\title{
Liberté et Sécurité en Europe : enjeux contemporains
}

Didier Bigo, R.B.J. Walker et chercheurs ELISE

\section{(2) OpenEdition \\ 1 Journals}

\section{Édition électronique}

URL : http://journals.openedition.org/conflits/2040

DOI : 10.4000/conflits.2040

ISSN : $1777-5345$

Éditeur :

CCLS - Centre d'études sur les conflits lilberté et sécurité, L'Harmattan

\section{Édition imprimée}

Date de publication : 1 mars 2006

Pagination : 103-136

ISBN : 2-296-00622-1

ISSN : 1157-996X

\section{Référence électronique}

Didier Bigo, R.B.J. Walker et chercheurs ELISE, « Liberté et Sécurité en Europe : enjeux contemporains », Cultures \& Conflits [En ligne], 61 | printemps 2006, mis en ligne le 17 mai 2006, consulté le 30 mars 2021. URL : http://journals.openedition.org/conflits/2040 ; DOI : https://doi.org/ $10.4000 /$ conflits. 2040

Ce document a été généré automatiquement le 30 mars 2021.

Creative Commons License 


\title{
Liberté et Sécurité en Europe : enjeux contemporains
}

\author{
Didier Bigo, R.B.J. Walker et chercheurs ELISE
}

1 1. Introduction

2 1.0. ELISE - European Liberty and Security: Security Issues, Social Cohesion and Institutional Development of the European Union - est un programme de recherche financé (pour une durée de trois ans à compter d'octobre 2002) par le Cinquième Programme-cadre de recherche et de développement de la Commission européenne ( $5^{\mathrm{e}} \mathrm{PCRD}$ ). Ce rapport présente les principaux résultats de la recherche, souligne les implications en termes de politiques publiques, et propose des recommandations ${ }^{1}$.

3 1.1. Le point de départ critique de ce programme est l'argument discutable selon lequel les traditions libérales et démocratiques des politiques modernes européennes peuvent toujours combiner aspirations à la liberté et aspirations à la sécurité. Cet argument se répète, alors que nous savons bien que ces deux valeurs ont entretenu, au cours de leur histoire, des relations pour le moins houleuses. A partir de la relecture de cette histoire, nous avons voulu comprendre les préoccupations récentes des citoyens européens en matière de sécurité, tout en gardant à l'esprit que celles-ci s'accompagnent du souci de ne pas porter atteinte aux libertés publiques, aux droits de l'Homme et à la cohésion sociale. Pour ce faire, nous avons essayé de replacer ces dilemmes contemporains dans un cadre plus large que les études de sécurité, en permettant à un large éventail de traditions et de disciplines universitaires de s'engager dans une recherche productive sur ces domaines communs de préoccupation.

4 1.2. De nombreux défis sont posés aux principes, aux institutions et aux pratiques sociales, à partir du moment où l'on veut à tout prix concilier liberté et sécurité. Certains de ces défis sont reliés à l'émergence de l'Europe en tant que nouvelle forme d'organisation politique. D'autres résultent des formes de développements économiques, culturels et technologiques identifiés sous le terme de "mondialisation ». Certains proviennent d'inquiétudes croissantes quant aux nouvelles formes de violence associées aux attentats du 11 septembre 2001, et aux différentes réponses à ces 
attentats. D'autres encore sont liés aux perceptions de la nature des Etats souverains et des relations qu'ils entretiennent.

5 1.3. Il a donc fallu revenir plusieurs siècles en arrière pour comprendre comment la relation entre liberté et sécurité s'est articulée dans l'Etat et dans le système international moderne. Outre cette analyse historique, nous avons étudié les changements structurels contemporains qui affectent les politiques sécuritaires européennes, et qui incluent, parmi d'autres aspects, le processus d'élargissement, les accords sur les frontières externes communes de l'Europe, et les nouvelles stratégies antiterroristes développées au niveau national, européen et international. Cela a impliqué l'élaboration d'une analyse critique de ce qui est en jeu dans l'idée apparemment retenue par le sens commun de la nécessité d'atteindre un juste " équilibre » entre sécurité et liberté.

6 1.4. Cette métaphore d'un équilibre parfait entre liberté et sécurité, comme chacune posée sur le plateau d'une balance, permet de cerner bon nombre de présupposés concernant la place de la violence légitime dans la vie politique moderne. Elle montre également un récit extrêmement trompeur des forces sociales, des pratiques institutionnelles et des principes juridiques à l'œuvre dans les sociétés démocratiques contemporaines. Cela est particulièrement vrai pour les questions liées à la liberté et la sécurité. ELISE a cherché à démêler les dynamiques - nombreuses, différentes et souvent conflictuelles - obscurcies par cette métaphore, pour offrir un compte rendu plus riche de ce qu'impliquerait un compromis entre les libertés établies et les principes de sécurité. Si des décisions difficiles doivent être prises, on ne doit pas se baser sur des métaphores d'équilibre, confuses et dépolitisantes, mais sur des questions de fond, comme le fait de connaître la signification des exceptions ainsi introduites par rapport aux attentes normales de liberté, d'égalité, de démocratie et d'Etat de droit dans la vie politique moderne.

7 1.5. ELISE a donc travaillé à partir de données techniques précises sur une politique de l'exception, remettant en cause l'hypothèse trop simpliste d'un besoin d'équilibre entre liberté et sécurité. Ces analyses ont mis à nu les limites et les racines intellectuelles des analyses de sécurité, fondées sur les traditions du réalisme politique; elles discutent des traditions légales soucieuses des limites de la règle de droit et les mises en récits historiques des sociétés libérales et démocratiques lorsqu'elles sont confrontées à des pressions les engageant à devenir plus illibérales et plus autoritaires. ELISE a donc d'abord cherché à examiner avec précision comment une nouvelle politique de l'exception se construit en réponse à des revendications relatives aux nouvelles formes d'insécurité. Le programme a ensuite voulu évaluer leurs implications plus larges. A partir de là, nous nous sommes intéressés aux conflits potentiels entre les libertés publiques et les droits de l'Homme, les mesures sécuritaires spécifiques, et les effets de telles ou telles mesures sur la construction socio-économique générale de l'Europe, ainsi que sur le conflit potentiel entre des mesures techniquement efficaces et des menaces de désaffection des groupes de population visés.

8 1.6. Les membres d'ELISE pensent que plusieurs des nouvelles réponses politiques constituent des remèdes tout aussi inquiétants dans le long terme que les maux auxquels elles veulent s'attaquer. En effet, elles ne sont pas sans rappeler la manière dont certaines perceptions de dangers immédiats ont mené à l'érosion du libéralisme et de la démocratie à d'autres moments de l'histoire européenne. En outre, un décalage croissant apparaît clairement entre les capacités organisationnelles vouées à la sécurité à l'échelle globale, et les ressources de plus en plus fragmentées pouvant être mobilisées pour maintenir les libertés dans des conditions démocratiques. 
a recherche menée principaux axes : un processus de redéfinition des concepts liés à la sécurité au niveau national, européen et mondial, et un processus de développement d'un cadre analytique permettant d'évaluer et de tester les réponses politiques aux futurs défis de sécurité - en particulier ceux qui impliquent des actes de violence politique officiellement labellisé «terroristes"-, et de construire des critères relatifs aux libertés publiques et à la cohésion sociale.

11 2.1. Dans ces deux axes, le programme ELISE s'est appuyé sur le très large aveu d'incapacité des récits traditionnels - d'une sécurité purement nationale définie en relation à une sphère extérieure ou internationale, à une arène purement nationale de liberté sous la loi d'Etats particuliers - à traiter des dilemmes contemporains. L'idée même d' « Europe ", comme label maladroit de la «mondialisation », tout comme la notion très litigieuse de "guerre au terrorisme» attestent de la nécessité d'une réflexion profonde sur les grands principes de base qui nous font nous percevoir comme des citoyens libres dans le cadre de la loi, et en sécurité au sein d'une existence collective qui ne soit pas diminuée par les prétentions des Etats souverains.

12 2.2. Le programme a donc été attentif à l'envergure des défis posés aux principes fondamentaux de liberté et de sécurité, qui résultent des développements contemporains. Il a fallu mobiliser des perspectives transnationales et transdisciplinaires, afin de comprendre la signification de ces défis pour la formation de notre capacité à juger les affaires complexes qui sont si clairement résolues dans la littérature universitaire conventionnelle. Le programme a donc dû s'accommoder de plusieurs formes de savoir issues des différentes traditions universitaires travaillant au sein d'ELISE, mais aussi des questions sur l'autorité de diverses agences revendiquant une capacité à émettre des jugements sur le sérieux de différents actes de violence et sur les conséquences qui devront nécessairement en découler.

13 2.3. Le fait de réunir dans un même débat, différents modes de pensée - d'un côté des discours de sécurité nationale et, de l'autre, des discours politiques et sociologiques sur la citoyenneté - illustre les difficultés des sujets auxquels l'équipe a dû faire face. A un autre niveau, la prise en considération de l'extraordinaire complexité des pratiques juridiques et administratives contemporaines - qui a rendu d'anciennes démarcations plus troubles - soulève des problèmes gênants, qui ne sont évoqués ni par les « internationalistes » ni par les « intégrationnistes ».

14 2.4. Les débats autour des notions et principes constituent une grande part du travail entrepris. Mais nous avons également voulu montrer comment des lois et des principes peuvent être façonnés par des «micro-pratiques". Ces dernières enclenchent des processus, établissent des pratiques, introduisent des règles de comportement (parfois par des accords fonctionnels). Ce qui n'était qu'une opportunité de court terme se transforme en routine bien enracinée, de même, des intentions louables en viennent assez fréquemment à générer des effets négatifs. L'un des objectifs principaux a été de replacer les enjeux des drames de ces cinq dernières années dans une perspective historique et comparative, sans perdre de vue les détails des pratiques et des événements. Ces derniers conduisent à de nouvelles approches et des réponses à la question de la relation entre liberté et sécurité - parfois sérieuses, parfois pas - et soulèvent souvent des questions fondamentales sur la responsabilité politique dans l'Europe contemporaine. 
3.0. En soulevant de telles questions sur les principes et les notions au sein d'arènes politiques particulières, l'accent a été mis sur trois objectifs majeurs : la compréhension des défis contemporains de la sécurité, la description des développements des politiques de sécurité, l'étude des conséquences de la modification de la relation entre liberté et sécurité.

17 3.1. Dans un premier temps, le projet a cherché à développer une meilleure compréhension des défis contemporains de la sécurité. A partir du grand nombre d'ouvrages parus depuis que les hypothèses sur la " sécurité nationale » et le " réalisme politique » ont perdu de leur crédibilité avec la fin de la Guerre Froide, ELISE a cherché à travailler avec une large communauté de chercheurs en science politique, sociologie et droit. Le programme a voulu penser aux implications des notions de «bonne gouvernance » dans les revendications de sécurité, et résister aux appels métaphysiques sur une " politique de puissance et de pouvoir » universelle par une évaluation attentive des techniques spécifiques d'exercice du pouvoir dans nos sociétés modernes. ELISE a également voulu évaluer le travail fait par le déploiement de conceptions spécifiques du terrorisme, afin de comprendre les implications des tentatives de constitutionnalisation des politiques sécuritaires, et, surtout, arriver à une réflexion sur les implications de la relation changeante entre des revendications de liberté et des revendications de sécurité à l'échelle européenne, ce qui implique de parler de l'Europe comme d'un lieu de légitimité politique fondé sur des appels à l'Etat de droit.

18 3.2. Dans un deuxième temps, ELISE a cherché à produire un compte rendu détaillé du développement des politiques de sécurité à un niveau national et européen, en particulier au lendemain d'événements comme ceux du 11 septembre 2001, et de leur impact sur la cohésion des sociétés européennes.

19 3.3. Dans un troisième temps, ELISE a cherché à identifier les premiers défis institutionnels auxquels les Etats membres et l'Union européenne sont confrontés. Ces défis sont nés de la modification de la relation entre liberté et sécurité dans des contextes à chaque fois extrêmement différents.

20

3.4. ELISE constitue donc un programme très large, combinant le sentiment important de besoin d'innovation conceptuelle et la volonté de s'attacher particulièrement à ce qui s'est passé en des endroits bien spécifiques, dans des arènes politiques, au niveau des contestations juridiques et des décisions du pouvoir exécutif. En cela, le programme ELISE a réaffirmé le besoin intellectuel de s'assurer contre la tentation d'un savoir spécialisé qui s'appellerait étude de sécurité, droit, criminologie ou libertés publiques. Car les plus grands défis viennent de plus en plus clairement des limites et des lacunes de ces champs d'expertise familiers.

21 3.5. ELISE est parvenu à travailler en dépassant certaines de ces limites, mais en reconnaissant que les différentes possibilités d'avenir européen impliqueront toujours des lignes de démarcation de toutes sortes, et que les frontières entre les demandes de liberté et les demandes de sécurité continueront à encadrer les possibilités de revendication d'une Europe qui est, d'une certaine façon, toujours libérale, démocratique, légitime et impérieuse. De plus, toutes les considérations de la relation entre sécurité et liberté ont un intérêt commun pour les frontières et les limites, ce qui a maintenu un niveau surprenant de compréhension et de communication entre différentes disciplines: théorie politique, sociologie, criminologie, relations 
internationales, analyse juridique et politique. Cet intérêt commun pour l'identification des problèmes s'est avéré plus efficace que les pseudo-tentatives d'harmonisation des techniques employées par les chercheurs les plus conventionnels.

clairement des discours de nombreux hommes politiques, d'une grande part des medias de masse, et d'une certaine forme de recherche universitaire fondée sur les récits nationalistes de la relation amis-ennemis. Toutefois, ces résultats semblent corroborés par un consensus émergeant d'un large spectre contemporain d'universitaires et d'opinion informée. Parmi les conclusions générales, deux ne semblent pas prêter à discussion et amènent à s'inquiéter des jugements prématurés sur la nouveauté de la situation présente. Les récits sur le « radicalement nouveau » peuvent trop facilement se transformer en récits sur le « radicalement dangereux ».

On ne peut raisonnablement considérer le 11 septembre 2001 comme un "événement sans précédent", qui aurait radicalement changé le visage du monde moderne, quand bien même ce fut un moment tragique. Cette date n'a pas marqué la naissance d'un nouvel âge du terrorisme, ou de l'hyper-terrorisme, ou du mégaterrorisme, ou encore d'un terrorisme de troisième type. La transnationalisation de la violence politique par des organisations clandestines est un long processus, dont les racines remontent aux mouvements de décolonisation des années 1950, aux prises d'otages et aux détournements d'avions par des tiers parties dans les années 1970, au développement des meurtres à distance via les technologies de bombes télécommandées dans les années 1980, à la radicalisation des conflits en Palestine, et à la résurgence d'attentatssuicides contre les casernes des armées française et américaine au Liban. Si nouveauté il y a, il s'agit plutôt de la combinaison nouvelle de formes d'action traditionnelles, et non pas, comme tant de récits officiels l'ont sous-entendu, d'une force d'un type nouveau combinant armes de destruction massive et organisations clandestines fanatiques et irrationnelles.

4.2. A partir des preuves disponibles, nous pouvons constater que les organisations clandestines contemporaines n'ont apparemment pas cherché à recourir à des armes de destruction massive. L'utilisation d'anthrax, par exemple, ne semble pas liée à al-Qaida, bien que les craintes relatives aux armes biologiques soient désormais largement répandues. Les liens entre l'Irak de Saddam Hussein, les armes de destruction massive et les organisations clandestines transnationales ont été fortement affirmés, mais n'ont, clairement, aucun fondement à ce jour. En revanche, nous pouvons suspecter que, depuis ces attentats, de nombreux professionnels de la politique ont une peur en commun : celle de voir disparaître leur revendication sur le monopole de la violence légitime au sein d'un territoire national spécifique. L'un des arguments de cette conception traditionnelle du monopole de la violence revient à considérer que la seule alternative à celui-ci constitue, en quelque sorte, un cauchemar apocalyptique, avec la perte de tout contrôle politique dans des situations de révolution, comme celle évoquée par Lénine ou encore comme la situation chaotique d'une grande partie de l'Europe à la fin de la Première Guerre mondiale. Une telle alternative a longtemps fait du monopole du pouvoir par un Etat moderne plus ou moins raisonnable le terrain manifeste d'un réalisme politique en construction, au lendemain des menaces révolutionnaires. Ainsi, cela revient-il à considérer l'Etat-nation moderne comme le seul terrain naturel de toutes les possibilités politiques. 
26 4.3. C'est ce spectre de l'anarchie qui semble de nouveau hanter les professionnels de la politique. Ceux-ci en viennent en effet à considérer l'Etat comme allant de soi, comme l'unique source d'autorité politique contemporaine et comme le lieu nécessaire de toutes les décisions relatives à la légitimité de la violence. Ce type d'alternative réapparaît à présent comme la peur d'un nouvel Armageddon préparé par de petits groupes de fanatiques, qui, au nom de la religion, usent d'armes de destruction massives prêtes à l'emploi : une combinaison, en quelque sorte, de la secte japonaise Aum et des ressources organisationnelles des réseaux d'al-Qaida.

27 4.4. Les professionnels de la politique se livrent sans conteste, à partir de données empiriques, à une surinterprétation superficielle de ces récits, ceux-là même qui décrivent un nouvel environnement menaçant et qui exigeraient un jugement plus circonspect. Quelle que soit l'explication à donner à ce phénomène, il semble évident que la peur de l'avenir devient un élément significatif de la vie politique contemporaine. Cette peur a été particulièrement accentuée par certains membres de l'administration américaine qui l'ont instrumentalisée dans le but de prendre l'avantage parmi certains électeurs, en faisant des attaques du 11 septembre 2001 une menace pour l'existence même des Etats-Unis. Dans cette optique, l'administration a généré des discours forts qui ont d'une certaine façon légitimé les revendications d'état d'urgence, le besoin de suspendre la politique menée habituellement, la nécessité de déclarer la guerre, et de se lancer dans une nouvelle confrontation entre amis et ennemis- bien que ceux-ci ne soient pas facilement repérables sur la carte des puissances en compétition dans le système international.

28 4.5. Les processus précis engagés ici sont sans aucun doute très complexes. La conséquence en a sûrement été la puissante tentative faite pour cultiver un ultranationalisme, basé sur un désir de vengeance. Cette tentative a requis la création de distinctions de plus en plus tranchées entre amis et ennemis, dans un nouveau monde soi-disant en perpétuels conflits inter-civilisationnels. Les dangers des excès rhétoriques dans ce contexte ont souvent été constatés. Mais n'oublions pas que la rhétorique peut s'avérer très efficace. Elle peut en effet créer des prophéties qui se réalisent elles-mêmes, lorsque des énoncés sur les menaces, dangers et terreurs en viennent à recouper des revendications de longue date au terme desquelles la seule alternative à l'Etat de sécurité moderne serait effectivement une sorte de chaos apocalyptique. De nombreux professionnels de la politique sont enclins à avoir ce mode de pensée, et se laissent facilement entraîner dans des discours pointant des dangers radicalement nouveaux. Pour porter un jugement plus sobre, il faudrait qu'une réelle attention soit prêtée aux continuités et à des ajustements relativement subtils, et qu'on tempère cette tendance qui transforme des événements comme ceux du 11 septembre 2001 en des moments clés dans la narration d'une apocalypse à venir.

4.6. Le 11 septembre, de par ses formes de violence dites «terroristes », ne constitue ni une rupture avec le passé, ni l'entrée dans une ère radicalement nouvelle. Il ne marque pas non plus de rupture dans les pratiques utilisées pour répondre à des «violences terroristes ". La référence à une condition de danger extrême, déclarée ou non, est certainement l'une des ressources les plus importantes à la disposition des gouvernements des Etats. Elle a une longue histoire de pratiques, tant par la ruse de la raison d'Etat que par la loi constitutionnelle. L'utilisation de mesures dérogatoires aux droits de l'Homme et aux droits à la vie privée a été déjà largement développée en Algérie à la fin des années 1950. Ces mesures se sont vues reproduites au Brésil, en 
Argentine, en Uruguay, au Chili et dans d'autres Etats d'Amérique latine, comme, d'ailleurs, dans des Etats libéraux tels l'Allemagne (contre Baader Meinhof), l'Italie (contre les Brigades rouges), l'Espagne (contre l'ETA), et, plus particulièrement, le Royaume-Uni (contre l'IRA en Ulster) et la France (après 1986).

4.7. Les politiques antiterroristes post- 11 septembre 2001 aux Etats-Unis font ellesmêmes suite aux propositions mises en place en 1999 et adoptées par l'administration Clinton. Ces mesures ont été façonnées par des adaptations constitutionnelles à des pratiques bureaucratiques existantes. Elles prolongent des tendances exprimées, notamment dans les réponses aux attentats perpétrés contre les Américains au début des années 1980 (au Liban et à Berlin), les frappes contre la Libye et, plus tard, la mise en place du Central Command au Pentagone. Les principaux débats en Europe et aux Etats-Unis depuis 2001, qu'ils traitent de la réorganisation et de la coordination des structures de renseignement, du Homeland Security, des services d'immigration et de naturalisation, des gardes frontières, ou de la priorité donnée aux mesures militaires, suivent les chemins tracés par des logiques plus anciennes.

31 4.8. Plus précisément, une série de mesures a été proposée au lendemain du 11 septembre, mesures qui avaient déjà été envisagées avant 2001, mais qui avaient été à l'époque rejetées, car jugées inacceptables dans leur principe. On peut donc véritablement s'interroger sur cette forme d'acceptation de ce qui avait été refusé auparavant, et surtout sur les raisons de ce changement.

4.9. L'adhésion à des logiques plus anciennes explique en partie pourquoi un certain nombre de politiques antiterroristes actuelles sont à ce point orientées vers la technologie et préoccupées par l'utilisation de la surveillance, de la biométrie, de la transnationalisation des informations des services policiers et de renseignement, et des demandes de collaboration accrue entre organisations de police sous la direction des services de renseignement américains. En ce domaine, la politique de l'Union européenne a été encore moins innovante que la politique américaine. Le cadre européen comprend un certain nombre de mesures portant sur les échanges d'information, la coopération policière et judiciaire, la sécurité des papiers d'identité, le blanchiment d'argent, le gel des biens et capitaux, et les principaux acteurs ont insisté sur la création d'instruments spécifiques comme le mandat d'arrêt européen - la mise en place de certaines institutions comme Eurojust, de nouveaux pouvoirs donnés à Europol, l'automatisation et l'accélération des procédures, le contrôle renforcé de l'Internet, la surveillance accrue des manifestations de masse, et le lancement de discussions routinières entre services de renseignement. Mais, après analyse, aucune de ces mesures ne peut être considérée comme une réaction rapide au 11 septembre ou à des pressions américaines ultérieures. Elles sont, dans leur ensemble, le résultat d'un ancien courant de propositions dont l'origine remonte au début des groupes Trevi et Schengen au milieu des années 1980. Ces propositions se sont vues opposer des résistances partielles à Amsterdam et Tampere, mais sont devenues la tendance dominante après Gènes.

33 4.10. Ainsi, une fois encore, il semble que la continuité ait été plus significative que l'innovation pratique, bien que les limites de ce qui est acceptable aient été clairement déplacées à la suite de l'ampleur de la violence des organisations clandestines. Une transformation significative dans l'articulation générale de la signification de la sécurité a également été opérée. Malgré un recours renouvelé à des rhétoriques nationalistes, les demandes en faveur d'une sécurité purement nationale ne monopolisent plus les discours professionnels ou publics. Avec la mise en place de réseaux institutionnels de contrôle, 
l'Union européenne a développé ce qui s'apparente à un appel et à une forme de sécurité globale, via un ensemble d'institutions et de procédures impliquées dans la gestion des flux de populations et de biens, de manière à protéger le bon fonctionnement du marché, de ses infrastructures humaines, institutionnelles et techniques.

4.11. Cette forme de sécurité reflète l'éloignement, souvent constaté, des Etatsprovidence du type de celui associé aux gouvernements nationaux et à la régulation économique néo-keynésienne. Elle témoigne également, par de nombreuses institutions formelles, de l'émergence de nouvelles formes et techniques de gouvernance. En ce sens, les concepts de sécurité et de protection sont associés au concept de prospérité économique, et, de ce fait, englobent toutes les menaces qui pourraient potentiellement mettre en danger les conceptions du bien-être fondées sur le marché. C'est pourquoi Gènes, symbole de la contestation du G8, peut être vu comme un tournant plus important que celui du 11 septembre 2001. Néanmoins, ce dernier a certainement changé nombre de relations de pouvoir et a permis aux Etats-Unis de devenir un acteur plus important "à l'intérieur même » des débats européens, encourageant ainsi des mesures plus coercitives et proactives, diminuant l'importance des garanties juridiques de l'individu, de la protection des données et de l'habeas corpus. La déclaration commune des Etats-Unis et de l'Union européenne de 2004 sur la lutte contre le terrorisme (The 2004 Joint EU-US Declaration on Combating Terrorism), en particulier sur la lutte contre le financement du terrorisme par la première troika ad hoc européano-américaine sur la prévention du financement terroriste, incluant les trois piliers, offre, de ce point de vue, un exemple important.

5. Cinq sujets de préoccupation liés à l'antiterrorisme

5.0. Il est nécessaire de mettre l'accent sur les continuités ainsi que sur les innovations dans les évaluations des violences politiques contemporaines dites «terroristes » et de leurs réponses. Cette constatation a émergé d'une analyse généalogique et multidimensionnelle sur les transformations de l'utilisation de la violence politique de la part des organisations clandestines et sur les stratégies antiterroristes. En pratique, ELISE s'est principalement concentré sur le second aspect. Il est pour l'instant quasiment impossible de savoir de manière sérieuse et fiable ce qui se passe dans les réseaux al-Quaida et quelles sont les situations qui y sont liées en Arabie Saoudite, au Yémen, en Indonésie, en Afghanistan, en Palestine et en Algérie. Les historiens nous en diront certainement plus. Cependant, dès à présent, il est possible d'évaluer la manière dont les stratégies antiterroristes ont tenté de comprendre les formes contemporaines de violence politique et d'y répondre. Dans ce contexte, cinq points au moins doivent retenir notre attention.

37 5.1. Premièrement, les principaux récits politiques portant sur al-Quaida et sur les organisations similaires tendent à reproduire, comme par mimétisme, les formes de savoir spécialisées et décontextualisées produites par les services de renseignement. Ces formes de savoir sont faussement libres, à la fois de toute responsabilité démocratique et des évaluations critiques effectuées par d'autres institutions. Le savoir produit par les services de renseignement a bénéficié d'une sorte de statut privilégié à cet égard. Cependant, ce statut dépend précisément de l'absence de critique ouverte, celle-là même qui constitue la condition sine qua non de la distinction entre savoir et dogme. Il n'est pas surprenant que les récits produits dans ces conditions aient été fortement influencés par les expériences des années 1970, conservant ainsi la même structure analytique, sans compter un fort déterminisme technologique. La plupart des 
" solutions " principales qui ont été trouvées sont en effet imprégnées de visions très anciennes. Elles témoignent même d'une résistance contre les analyses plus complexes et plus subtiles dont l'influence s'est développée au milieu des années 1990. En d'autres termes, les complexités et subtilités pourtant nécessaires ont été abandonnées au profit de simplicités plus anciennes mais plus limpides, qui servent par la suite de base aux obsessions - non dénuées d'esprit critique - pour les innovations technologiques qui, à en croire certains, suffiraient à régler les problèmes politiques.

5.2. Deuxièmement, les demandes de nouvelles formes de sécurité ont commencé à agir comme des demandes de nouvelles formes de gouvernement - de "gouvernance", pour reprendre le terme de ceux qui comprennent la portée limitée des gouvernements étatiques, ou de "gouvernementalité ", pour rejoindre ceux qui partent du principe que les individus sont faits pour se gouverner eux-mêmes plutôt que d'obéir aux ordres d'un gouvernement. Lorsque les nouvelles technologies sont traitées comme de simples ajouts à des processus politiques établis, les techniques et stratégies spécifiques peuvent générer de nouvelles formes de contrôle social. Les formes de responsabilité associées aux formes modernes de gouvernement sont progressivement subverties par les procédures de contrôle social. Dans ce contexte émerge alors un besoin accru d'attention pour ce qu'il était auparavant convenu d'appeler des "conséquences involontaires de l'action politique». Celles-ci ont finalement bénéficié d'un vide politique, car elles ont tenté de fournir une sécurité via des technologies.

5.3. Troisièmement, l'attachement à la valeur donnée aux nouvelles technologies et à l'idée d'un chaos quasi apocalyptique comme seule alternative au monopole de la violence dans un territoire spécifique, articule les récits sur la sécurité autour d'une capacité à contrôler le futur. De nombreuses préoccupations obsolètes réapparaissent alors, notamment au sujet d'un crédit exagéré donné aux scénarios catastrophes, aux hybris, ou aux conséquences involontaires. Il y a aussi des raisons de s'inquiéter de l'étendue des demandes de sécurité pour contrôler les populations, en lien non seulement avec les dangers qui ont au moins des références tangibles et concrètes, mais aussi avec les idées spéculatives, abstraites et métaphysiques sur des futurs qui ne peuvent être qu'imaginés - et ce, à partir d'idées dépassées, sur ce que les ennemis sont censés faire aux amis. Les dites doctrines de guerre préventive et de police proactive expriment toutes deux ces tendances pour le moins gênantes. A cela s'ajoute le fait que les professionnels de la politique et les membres des services de renseignement et de sécurité ont été de plus en plus en compétition pour évaluer ce qui s'est passé et ce qui va se passer. Au niveau de l'Union européenne par exemple, le Conseil évalue les menaces à partir des travaux du Centre de Situation dont les évaluations diffèrent de celles d'Europol dans la mesure où les militaires et policiers ont des visions différentes dans la manière d'analyser ce qui est menaçant.

40 5.4. Quatrièmement, les connaissances émanant des services de renseignement et de sécurité bénéficient souvent d'un statut privilégié par rapport à celles provenant d'autres acteurs politiques. C'est une conséquence de la façon dont les médias de masse prêtent attention aux voix des élites spécifiques. L'analyse des déclarations d'acteurs politiques nationaux en France et au Royaume-Uni publiées dans la presse entre septembre 2001 et juin 2003 montre, par exemple, que la défense des formes d'état d'urgence est largement couverte par la presse, qui est, quant à elle, soutenue par un nombre restreint d'acteurs politiques. Mais elle n'obtient pas le soutien ouvert de la majorité des acteurs politiques nationaux. De l'autre côté, les droits de l'Homme sont moins défendus par les leaders politiques que par le public. 
41 5.5. Cinquièmement, et comme l'illustre tout particulièrement une étude du cas italien, les revendications sécuritaires engendrent des abus considérables, par l'imposition des catégories totalisantes de l'ami et de l'ennemi à de nombreuses autres formes de différence politique. Dans le cas italien, les revendications de différence sont principalement liées au rôle du pays au titre de «limite-sud» de l'Europe. Que ce soit dans les discours journalistiques, la presse, les débats politiques, ou les connaissances générales des requérants italiens, la soi-disant « invasion » des « migrants illégaux » est souvent traduite par des termes synonymes de «dangers terroristes». Deux facteurs locaux agissent dans ce cas: la criminalisation traditionnelle des migrants comme étrangers dangereux, et la prétendue faiblesse des frontières italiennes face aux infiltrations terroristes. Bien qu'il n'y ait aucune preuve de lien entre terrorisme et migrations illégales, il est devenu normal aujourd'hui de chercher les terroristes parmi les migrants uniquement, en particulier parmi ceux qui viennent des pays musulmans. Il est également devenu normal de s'acharner au-delà du raisonnable sur certains suspects, ce qui a mené à plusieurs procès au cours desquels des personnes ont été accusées avant d'être finalement acquittées. La construction sociale des migrants comme terroristes potentiels a été néanmoins efficace. Elle a permis une stratégie d'internement et d'expulsion «facile", mais a finalement engendré l'accusation des autorités italiennes par les Cours internationales pour violations des droits de l'Homme.

6. Sécurité, liberté et défis à la démocratie

6.0. Ces cinq sujets de préoccupation attirent donc l'attention sur le statut incertain des formes contemporaines de démocratie. Comme nous l'avons remarqué, les préoccupations récentes sur la sécurité ont encore développé le très ancien dilemme de la défense de la démocratie par des moyens de coercition exceptionnels, sans toutefois le résoudre.

6.1. L'analyse de la presse italienne montre, de manière presque symptomatique, l'absence de tout débat significatif sur les implications des politiques sécuritaires contemporaines. Ceci est étroitement lié à la configuration spécifique du champ politique national, et s'explique en partie par le fait que les dispositions juridiques administratives et légales étendues aux terroristes étaient déjà en vigueur pour le crime organisé. Il suffit souvent de mettre des éléments supplémentaires à l'intérieur d'un encadrement déjà chargé du maintien de la sécurité contre la terreur pour couper court à toute discussion.

6.2. Au niveau de l'Union européenne, l'analyse des débats au Parlement européen sur la lutte contre le terrorisme (entre septembre 2001 et juin 2003) suggère également qu'avant le 11 septembre 2001, les questions liées à l'invocation de mesures d'urgence étaient rarement considérées comme liées à des attentats terroristes. Lorsque ces questions se sont retrouvées sur le devant de la scène, au lendemain de ces attentats, le besoin de dérogations d'urgence n'a été finalement défendu intellectuellement que par une petite minorité de députés européens. La majorité des parlementaires a mis l'accent sur les principes des droits de l'Homme. Les représentants des institutions européennes ont, eux, refusé de trancher et ont utilisé la métaphore de la balance, de l'équilibre, dont nous avons parlé précédemment. Bien que les représentants du Conseil n'aient ainsi jamais défendu ouvertement la nécessité de règles d'urgence, mais plutôt celle des droits de l'Homme, le sentiment dominant a néanmoins favorisé la thèse, apparemment modérée mais en réalité illibérale dans sa pratique, selon laquelle un «équilibre » doit être trouvé entre sécurité et droits de l'Homme et que, dès lors 
certains de ces droits doivent s'effacer devant les impératifs de sécurité. Les déclarations explicites des représentants de la Commission sur les risques d'atteintes aux droits civiques et aux libertés publiques, compensés immédiatement par l'invocation des impératifs de sécurité collective ont renforcé le soutien à cette position. Ainsi, au nom de l'équilibre et non du droit à l'exception, la possibilité de l'adoption de règles d'urgence comme mesure nécessaire à la protection de la sécurité interne des pays européens et du reste du monde a été acceptée.

6.3. Une des réponses techniques apportées aux récentes attaques terroristes, qui a eu les plus lourdes conséquences est la tentative de fermer les frontières avec la volonté :

47 - de créer un Homeland Security européen (même lorsque la devise principale de la gouvernance européenne insiste sur la nécessité de garder les marchés ouverts et de faciliter la liberté de circulation) ;

48 - d'introduire de nouvelles technologies de traçage des individus ; filtrer de plus en plus les entrées de personnes ;

49 - d'incarcérer les suspects potentiels ;

50 - de recourir aux militaires et au renseignement pour maintenir l'ordre à l'intérieur d'un pays ;

51 - de défendre la nécessité de mesures dérogatoires au nom d'un futur potentiellement cataclysmique.

52 Ces mesures ne sont pas nouvelles et ont déjà été empêchées dans les années 1970 par l'émergence d'une culture politique qui a célébré les droits civiques des citoyens et des étrangers, et par le développement d'une législation garantissant la protection des données. Mais ces mesures ont ici triomphé et suscité peu de réactions. Il y a un lien tacite mais évident entre l'analyse sur les stratégies contre-subversive et de sécurité nationale des puissances coloniales et néo-coloniales (R. Trinquier, F. Kitson, l'Ecole de Panama) et la stratégie de sécurité intérieure actuelle. Cela permet peut-être d'expliquer pourquoi certains acteurs en sont venus à justifier des interrogatoires lourds et prolongés - qu'ils veulent distinguer de la torture en évoquant un « moindre mal » - à l'image de ceux utilisés au cours des « guerres sales » d'Algérie, de Birmanie et du Vietnam. Cela a créé des luttes au sein même des différentes forces armées, certains corps étant révoltés par ces pratiques professionnelles souvent confiées à des "spécialistes ", et ils ont été les premiers à rejeter ces formes de justification qu'ils n'ont que trop bien connues auparavant.

6.4. Le succès des discours réintroduisant un état d'esprit de guerre à l'intérieur même d'un pays, ou visant à la justification d'une diminution des libertés publiques au nom d'une certaine urgence, est fortement lié aux luttes entre professionnels de la sécurité au niveau transnational. Les recherches d'Alessandro dal Lago sur la généalogie des stratégies de prévention et leurs effets sur les régimes contemporains le montrent clairement. De même, Didier Bigo montre comment le champ transnational des professionnels de la sécurité s'est organisé en accord avec les divers discours de sécuritisation, c'est-à-dire, non pas en relation a un seul discours sécuritaire qui soit en profond désaccord avec les revendications de liberté, mais en relation à un large nombre de discours sécuritaires en compétition entre eux.

6.5. Dans ce contexte il est utile de rappeler que pendant la Guerre Froide, les avis des professionnels de haut rang et des généraux, étaient toujours issus des analyses des stratégistes, des spécialistes de la dissuasion, et non de celles des spécialistes des guerres asymétriques, des guerres périphériques ou des conflits de basse intensité tels 
qu'ils étaient appréhendés à cette époque. Mais, après 1979 et la révolution iranienne, et, plus encore, après la fin de la bipolarité et de la première guerre du Golfe, les spécialistes des guerres asymétriques étaient de plus en plus pressés de passer au niveau supérieur, arguant qu'ils traitaient, eux, de véritables guerres et du terrorisme. Le 11 septembre 2001 a, depuis, renforcé leur influence rhétorique. De façon plus alarmante, tout du moins en relation à la compréhension des frontières et des limites restée longtemps si centrale dans les conceptions modernes de la possibilité politique -, cet éloignement de la stratégie (classique) a mené au déploiement de policiers au-delà des frontières des juridictions nationales. Les forces de police ont en effet été tentées de transposer leurs technologies de maintien de l'ordre national à l'extérieur, au nom de la consolidation de la paix. Cela a généré, comme on pouvait s'y attendre, une concurrence à l'étranger avec les militaires.

6.6. Les luttes pour la maîtrise et la réduction de la violence ont été importantes au Kosovo, en Afghanistan et en Irak, entre autres, ainsi qu'au sein de nombreux pays développant des politiques de gestion des immigrés et des citoyens d'origine étrangère. Ces luttes, peu surprenantes, ont créé des confusions sur le statut de la guerre, et sa comparaison avec les luttes internes et les conflits de classe qui traversent la société. Il ne fait aucun doute que la thématique de «l'ennemi de l'intérieur ", ou plus exactement de «l'ennemi furtif » a toujours été présente dans les discours et les pratiques des services de renseignement. Toutefois, depuis le 11 septembre 2001, elle occupe une place prépondérante pour de nombreux professionnels de la politique. Ceci soulève de nombreuses questions: avons nous une guerre à l'intérieur de nos société? Quel est le statut de «l'urgence nationale»? Dans quelle mesure pouvons nous considérer que les attaques terroristes sont une menace à la survie de l'Etat-nation? Le niveau d'autorité de l'Union européenne assume-t-il une protection et une promotion globales de la liberté (pour l'individu comme agent, la protection des données et la liberté de mouvement, l'aviation civile, ainsi que pour les Chartes comme régime général de protection) alors que des technologies de surveillance sont de plus en plus utilisées pour lutter contre des menaces spécifiques? Face à de telles questions, les différentes bureaucraties proposent des réponses différentes, chacune très proches de leurs intérêts propres.

6.7. Le fait central est que la sécurité, sans doute l'aspect le plus contesté du politique, en est venue à saturer tout discours politique. Il s'agit d'une arène de lutte par excellence, d'un champ de bataille sur lequel s'applique un sens particulier du politique. De ce point de vue, le fait que la sécurité soit d'actualité est donc le résultat d'interactions sociales, soutenues par ceux dont les intérêts sont en jeu, et dont le but est d'obtenir le mandat politique approprié et les ressources nécessaires à l'action visée. Au risque de simplifier à l'extrême, mais pour insister sur un point essentiel, nous pourrions dire que deux logiques discursives concurrentes s'affrontent aujourd'hui. D'un côté, on a la police et le judiciaire, qui conviennent que le terrorisme est un crime, et même un crime contre l'humanité, et qui s'opposent à l'argument voulant que le terrorisme soit une guerre au sein de la société. De l'autre côté, il y a les services de renseignement et les partisans de la guerre asymétrique, qui défendent l'idée d'une guerre au terrorisme à mener au sein de la société. Ce dernier argument ne doit pas être sous-estimé et devrait résonner tout particulièrement chez ceux pour qui l'histoire des luttes et résistances contre tant d'autres formes d'illibéralisme est familière. En effet, cet argument jette les bases d'une politique de l'exception et d'une diminution de l'Etat de droit.

7. Une nouvelle politique de l'exception 
58 7.0. De l'Anti-Terrorism, Crime and Security Act en 2001, à la loi sur la Prévention du terrorisme (Prevention of Terrorism Act) adoptée en 2005, pour ne prendre que l'exemple $\mathrm{du}$ Royaume-Uni, les changements législatifs et certaines pratiques administratives en Europe ne témoignent que trop d'une politique de l'exception et de l'utilisation d'une rhétorique du danger permanent. La spatialité et la temporalité qui déterminaient jusqu'à présent la capacité à déclarer l'exception de manière souveraine ont changé. Certains analystes vont même jusqu'à suggérer que le monde entier s'est internalisé et qu'une certaine capacité à proclamer des exceptions s'est généralisée. D'autres considèrent que la vie politique contemporaine est devenue plus complexe. Les deux positions se sont exprimées dans le programme ELISE, comme dans les débats plus généraux sur le terrorisme, la mondialisation, l'unilatéralisme et l'impérialisme. Toutefois, les appels contemporains à une guerre au terrorisme se sont déjà traduits au-delà des limites spatio-temporelles de l'Etat moderne souverain coexistant dans un système d'Etats souverains.

59 7.1. Les conséquences de cet excès se font ressentir dans les tensions des régimes libéraux démocratiques qui se considèrent en état de guerre permanent, plus qu'en état permanent de "paix démocratique libérale », état pourtant si célébré il y a dix ans à peine. La généralisation des déclarations à propos d'un état d'urgence comme moment déterminant de la vie politique soulève des questions essentielles au sujet des fondements de l'autorité légitime, de la capacité démocratique des individus à contester les revendications d'un Etat et ses articulations de souveraineté singulière au sein d'un territoire particulier, et enfin, de la possibilité même de l'action politique. Les pratiques sécuritaires fonctionnent en partie, et plus que tout autre sphère de la vie politique moderne, en cherchant à se protéger du fonctionnement normal de la contestation politique. Elles revendiquent en même temps leur capacité à protéger une sphère dans laquelle la contestation politique reste possible. En conséquence, porter son attention sur la mise en œuvre de pratiques sécuritaires, et particulièrement sur des législations antiterroristes, revient à s'engager dans l'action politique en relation non seulement aux droits, mais aussi à la contestation des revendications sur des conditions dans lesquelles l'autorité politique est aujourd'hui considérée comme légitime. Toute tentative visant à exclure les pratiques sécuritaires de la contestation politique dirige nécessairement l'attention vers la possibilité et les limites mêmes de l'action politique, particulièrement pour ce qui relève du judiciaire et de la société civile.

60 7.2. Le cliché selon lequel les gouvernements tendent à justifier leurs politiques sécuritaires par la nécessité de protéger leurs citoyens est le plus largement répandu dans l'analyse politique. De plus, et à la suite des travaux de Michel Foucault, la protection des populations revient nécessairement à protéger des catégories spécifiques de population à l'exclusion d'autres. Comme la majorité des recherches entreprises dans le cadre d'ELISE le suggèrent, la redéfinition des frontières portée par les nombreuses revendications sécuritaires contemporaines a généré le ciblage de catégories spécifiques de populations, selon des critères culturels et raciaux.

61 7.3. Les libéralismes traditionnels ont pendant longtemps distingué certaines catégories de population, à partir de considérations sur leurs capacités à gérer leur propre liberté. Mais ils ont aussi conçu des stratégies d'intégration sociale dans un espace national : au sein d'une sphère publique communicative, sur le fondement de prétentions égales aux droits civiques, par le biais de politiques d'Etat providence et des principes d'une justice distributive. Avec les réarticulations contemporaines des politiques d'exception, 
fonctionnant autrement que sur la base des frontières de ces Etats dans lesquels des pratiques d'intégration pourraient se cultiver, la différence, l'altérité et l'exclusion menacent de devenir le fondement à partir duquel s'organisent et se légitiment les relations politiques. Cela a des implications sur la façon dont les sociétés libérales répondent aux appels à la diversité, sur la façon dont les individus sont considérés, avec la pries en compte de leur identité culturelle et raciale, et sur la façon dont de nombreux antagonismes de longue date, entre principes libéraux et pratiques démocratiques, en arrivent à un point de rupture.

7.4. En effet, l'un des éléments clefs des discours sur un nouvel environnement sécuritaire a été ce retour à certaines traditions intellectuelles classiques qui ont cherché à penser ce qui se passe à la limite de la vie politique contemporaine pour justifier des mesures dérogatoires et la routinisation qui s'ensuit. Thomas Hobbes est sans doute le penseur le plus important dans ce contexte, pas du fait de son cynisme bien connu sur la nature humaine et la qualité anarchique de la vie entre individus libres et égaux, mais par la manière dont il a traité du pouvoir souverain capable de constituer une autorité juridique et de définir les limites de cette autorité. La réputation de Carl Schmitt, le théoricien constitutionnel allemand définitivement terni par ses tendances fascistes, est encore plus sombre. Il garde paradoxalement pour certains la crédibilité d'un penseur sérieux. C'est particulièrement en relation à Schmitt qu'on peut retrouver les origines de la réflexion contemporaine sur ce que cela signifie de traiter la souveraineté comme une capacité à faire des exceptions. Des courants de pensée similaires se sont intéressés à la tendance des sociétés libérales et démocratique à dégénérer en diverses formes d'autoritarisme.

7.5. La principale raison de l'intérêt que suscite encore ce genre de penseurs est qu'ils éclairent le fait que les sociétés - même les plus libérales - sont loin d'être immunisées contre des pratiques illibérales, et que les sociétés les plus démocratiques ont un potentiel autoritaire. De manière conventionnelle, l'autoritarisme et l'illibéralisme sont considérés comme des conditions-limites, c'est-à-dire comme des « démons » qu'on ne peut tolérer que dans des situations d'urgence et de guerre, lorsque les normes de conduite habituelles doivent être suspendues de manière à assurer la survie même de l'Etat. On constate ainsi que la capacité à décider de où et quand des exceptions doivent être faites, et à partir de quand les conditions-limites ont été atteintes rejoint la capacité de décider de quand et comment les appels à la liberté doivent céder la place à des appels à la sécurité.

7.6. Certains s'intéressent à ces penseurs car ils offrent un moyen efficace de justification de la nécessité de suspendre les libertés publiques face au danger. Telle est la dynamique qui s'exprime dans les traditions du réalisme politique venant influencer la plupart des discours sur la sécurité nationale. Telle est également la dynamique reproduite dans de nombreuses perceptions contemporaines de la menace terroriste. D'autres s'intéressent à ces penseurs car ils montrent comment un attachement sans complaisance aux Etats-nations souverains mènera probablement aux nécessités extrêmes demandées à la limite. Une situation à laquelle, selon eux, il faut résister par une compréhension claire de la facilité avec laquelle les libertés peuvent être détruites au nom d'un état d'urgence. Une grande partie de l'imaginaire politique qui a donné naissance à l'Union européenne vient justement d'une telle révulsion contre l'exceptionnalisme nationaliste qui prospérait dans les années 1920 et 1930. 
7.7. L'Europe est à présent assaillie d'appels en faveur de nouvelles formes d'exceptionnalisme, qui ne sont pas associées à des termes nationalistes mais qui constituent des demandes pour que des lignes soient tracées entre les possibilités de liberté et les nécessités de sécurité : «l'équilibre doit pencher vers la sécurité et non vers la liberté», "une exception doit être faite», ou encore: "les normes doivent être suspendues ", « les libertés doivent être réduites ». Dans une dynamique inverse, nombre de résistances à l'imaginaire unilatéral développé par l'administration Bush viennent du jugement selon lequel tout Etat qui se considère comme une exception risque toujours de promouvoir ses propres valeurs comme étant universelles et non internationales, et, en conséquence, de simplifier le monde en catégorie binaires, de célébrer l'analyse de scénarios catastrophes, et de créer un climat de suspicion détruisant la cohésion sociale au nom du patriotisme.

7.8. Les traditions classiques de pensée militaire ont longtemps affirmé que la capacité à décider des exceptions est une affaire des plus sérieuses. Un certain nombre d'analyses sur les problèmes sécuritaires contemporains ont perdu de vue ces traditions et se sont trop limitées à considérer toute menace, existante ou prédite, comme une preuve que les limites ont été atteintes, que l'état d'urgence existe, que les libertés doivent être restreintes, et les normes, suspendues.

7.9. Un tel raisonnement extrémiste n'est pas simplement dangereux, il est également inutile. Il ne trouve ainsi peu ou pas de soutien au sein des communautés universitaires compétentes. Il est plutôt le produit de circonstances spécifiques et identifiables impliquant le comportement de professionnels de la politique et la relation qu'ils ont avec la sécurité et les services de renseignement. Certains acteurs ont été capables de favoriser leurs intérêts en transformant une situation flexible avec plusieurs issues possibles en un «fait accompli ». L'un des grands paradoxes de notre époque a été que la sécurité s'est transformée, passant de sujet d'incertitude et de contingence, de jugement prudent sur l'imprévisible, d'un régime de responsabilité, à une capacité professionnelle et institutionnelle à savoir, avec certitude et avec tous les avantages de la science et de la technologie, qui est l'ami et qui est l'ennemi.

7.10. Ainsi, la décision d'envahir l'Afghanistan comme réponse appropriée à al-Qaida, celle de rendre prioritaire le Pentagone et l'industrie de la défense, celle de construire une Homeland Security, et celle d'éviter l'approche judiciaire et de maintien de l'ordre adoptée par l'Espagne après le 11 mars 2004 ont été façonnées par une vision unilatéraliste du monde spécifique, voire idiosyncrasique. Ces réponses n'ont pas été les seules possibles. Ceux qui se spécialisent dans les demandes d'un ordre politique international ou multilatéral leur ont en effet résisté. Mais ces décisions ont été prises, et leurs conséquences ne cessent de se déployer. Les sentiments initiaux de solidarité avec les victimes du 11 septembre 2001 ont laissé place à une suspicion et des ressentiments profonds sur la manière dont un acte de terrorisme a été amplifié, dans un premier temps, en un état d'urgence nationale, et, par la suite, en un raisonnement pour la restructuration complète de grandes parties du monde, et ce, à l'encontre des principes établis de l'ordre international. Il y a bien entendu une possibilité quasi infinie pour les désaccords sur les jugements et les décisions en œuvre au cours du déploiement de ce processus. Toutefois, deux points importants ne doivent pas être oubliés. Dans un premier temps, ces décisions spécifiques ont été générées par une politique de l'exceptionnalisme impliquant des appels à l'état d'urgence, des revendications qui ont été si « faciles » et si efficacement déployées dans un but qui n'a 
pas été uniquement la restriction des libertés mais aussi la justification d'une tentative de réécrire le fondement entier de l'ordre politique international. Dans un second temps, d'autres réponses ont été possibles, mais leur possibilité même a été rapidement éradiquée par des conceptions sur la nécessité militaire, des prétentions à un savoir légitimant la déclaration de guerre. Mais de graves dangers émergent lorsqu'on donne la capacité à des agences et des professionnels spécifiques de nous dire à quel moment la nécessité doit prendre le dessus sur nos vies et nos libertés.

69 7.11. L'une des manifestations les plus inquiétantes de la façon dont ces choix politiques ont été faits a été la volonté extraordinaire de traiter les "ennemis» comme des inhumains, si l'on considère que la fabrication des ennemis s'est faite via des appels à la défense de la civilisation. Abou Ghraib et Guantanamo sont des exemples significatifs, tout comme la volonté de cibler des populations entières comme des ennemis potentiels, si l'on considère les difficultés pour distinguer les "terroristes» des "civils», et l'indifférence avec laquelle l'échelle des dommages civils en Irak a été perçue dans de nombreux milieux. Aussi troublantes soient ces expressions, nous ne devrions pas oublier qu'elles expriment des tendances profondément ancrées, qui touchent le cœur de la structure des démocraties libérales modernes.

70 7.12. L'unilatéralisme de la première administration Bush est peut-être derrière nous. On envisage désormais une plus grande coordination avec l'Union européenne, une plus grande accentuation sur le maintien de l'ordre et du pouvoir judiciaire. Les débats déjà vifs sur la relation entre les Etats-Unis et l'Union européenne vont, sans aucun doute, se poursuivre et s'intensifier. Dans ce contexte, il est très important que les questions sur le choix des meilleures politiques de sécurité communes ne se transforment pas en débats sur les meilleures formes de coopération, sans que soit sérieusement pris en compte l'impact d'une telle coopération sur les libertés publiques. C'est important et pas seulement parce que les Etats-Unis conservent leur position hégémonique, si ce n'est unilatérale, mais plus encore parce que le phénomène de coopération implique plus une mondialisation effective des techniques et des agences concernées par la surveillance des personnes en mouvement, qu'une relation traditionnelle entre les parties. Les appels en faveur d'une coopération accrue entre agences de sécurité et services de renseignement mettent en jeu la construction de nouvelles manières de façonner les sociétés et leurs possibilités - ou leurs impossibilités - démocratiques.

71 7.13. Quelle démocratie voulons-nous aujourd'hui? Quelle liberté sommes-nous en train de sacrifier au nom de libertés futures et au nom de l'éradication des dangers terroristes? Autant d'interrogations mises de côté depuis bien longtemps. En effet, la question du besoin de sécuriser la démocratie, et celle de savoir si la démocratie reste une aspiration politique viable dans les conditions contemporaines sont à peine discutées au sein des élites ou dans les médias de masse, qui pensent sans doute que leur place dans le monde est plus ou moins acquise. Cela est sûrement lié à la manière dont l'industrie de la sécurité, ceux qui s'intéressent à la coopération économique entre membres de l'Union européenne et ceux qui sont en concurrence avec l'industrie américaine ont été capables de rendre leurs discours prioritaires par rapport à ceux engagés dans des débats sur les libertés publiques et la cohésion sociale. Néanmoins, d'un point de vue juridique, la liberté et la cohésion sociale sont intimement liées, à la fois au contrôle juridique des systèmes de pouvoir complexes et divers, et aux moyens politiques et administratifs pour la mise en œuvre de provisions légales sur des thématiques d'immigration. Tant que ces sujets seront envahis par des questions, 
jugées nécessaires par certains, de coopération sur des sujets sécuritaires ou économiques, les énergies démocratiques ne cesseront de s'affaiblir. Beaucoup de recherches menées dans le cadre d'ELISE suggèrent que la liberté et les droits fondamentaux de l'Homme ont en effet été compromis par l'omniprésence des discours sécuritaires. De surcroît, ces discours ont rendu encore plus difficile le fait de ne pas assimiler les appels à la démocratie à de simples figures rhétorique qu'on utiliserait pour introduire une discrimination entre Nous et les Autres.

72 7.14. De nombreuses autres dynamiques au sein de l'Union européenne soulèvent des inquiétudes sur les directions possibles de la coopération entre Etats-Unis et Union européenne. Bien que la crainte du terrorisme et de ses liens avec les armes de destruction massive ait été moins importante en Europe, on a tout de même vu une résurgence des formes d'insécurité générée au milieu des années 1980 au nom d'une lutte contre la liberté de circulation et de la peur de «l'invasion » de l'Ouest par les migrants. En Europe, comme aux Etats-Unis, des discours efficaces ont rattaché le terrorisme à la migration et à l'islam. De nombreux hommes politiques, dans un style classique populiste, ont déployé ce genre de discours pour se faire réélire, créant ainsi une mentalité de "siège ", en particulier dans les endroits où les individus n'ont que très peu de contacts avec d'autres populations et d'autres lieux, et craignent ainsi le mode de vie des Autres.

73 7.15. Bien que l'Union européenne ait, dans l'ensemble, essayé de construire une vision alternative de la sécurité par le projet d'élargissement, en incluant les Balkans par exemple ; bien qu'elle ait réussi à éviter des préjudices excessifs contre les musulmans ; bien qu'elle ait privilégié une coopération judiciaire et policière à l'échelle mondiale comme solution principale contre la violence politique; bien qu'elle ait essayé d'améliorer la stabilité économique au Moyen-Orient, certaines institutions ont usé d'une toute autre stratégie, reproduisant au niveau européen les hypothèses et procédures de leurs partenaires américains. Certains acteurs au sein de la Justice and Home Affairs ont, par exemple, tenté d'influencer le programme en vue de davantage de contrôles pour les migrants, au nom de la lutte contre le terrorisme et le crime organisé. Ils ont criminalisé les migrants et sécurisé les demandeurs d'asile. Ils ont considéré qu'ils étaient en guerre contre le terrorisme, et ont étendu leur lutte contre les migrants illégaux et les musulmans radicaux, en particuliers les imams.

\section{Les défis à l'Etat de droit}

8.0. Toutes ces préoccupations sont, dans une certaine mesure, distillées dans les tensions qu'on peut observer entre la classe politique et le judiciaire, en particulier pour le respect de l'Etat de droit. Le nombre croissant de détentions illimitées de personnes suspectées d'activités terroristes a été au cœur des discussions à la fois aux Etats-Unis, en relation aux citoyens étrangers détenus indéfiniment dans les bases américaines de Guantanamo, Cuba, et au Royaume-Uni, en relation à la 2001 AntiTerrorism, Crime and Security Act, qui autorise la détention indéfinie de citoyens étrangers, arrêtés et détenus dans la prison de Belmarsh, principalement. ELISE a examiné ces tensions en détail, en se concentrant particulièrement sur la détention illimitée, l'Etat de droit et le rôle des juges face aux pouvoirs exécutifs du gouvernement et des administrations.

76 8.1. Selon cette analyse, l'Etat de droit a été invoqué comme principe capable de compenser le faible contrôle démocratique des processus législatifs et exécutifs dans la protection du droit à la liberté des individus. Les conditions nécessaires à ce rôle 
controversé du judiciaire incluent la possibilité d'un accès aux Cours supranationales, en particulier la Cour européenne des droits de l'Homme, mais aussi la Cour européenne de Justice (CEJ). Cet aspect essentiel constitue un mécanisme qui permet aux juges de se sentir moins dépendants des législations et des corps législatifs nationaux. De plus, on demande aux juges d'appliquer le droit supranational dans le domaine national par le corps législatif. Ceux-ci sont donc protégés de toute accusation d'usurpation du rôle du corps législatif. La CEJ par exemple s'est servie des principes des marchés libres spécifiés dans les traités de la Communauté européenne, c'est-à-dire la liberté de mouvement dans le sens d'un accès au marché unique, comme contrepoids aux affirmations de l'intérêt général spécifiées en des termes nationaux. La position de l'Etat de droit comme principe organisateur au sein de l'Union européenne explique, d'une certaine manière, les différences entre la détention illimitée dans la prison britannique de Belmarsh et le cas des bases américaines dans la baie de Guantanamo.

8.2. Cette lutte autour de l'Etat de droit au niveau européen est cruciale. Cela ne dénigre et ne sous-estime toutefois pas le fait que nos sociétés aient réagi avec une certaine force aux utilisations de techniques antisubversives très coercitives, en particulier dans les années 1950 et 1960 en France et en Europe du Sud, et, plus récemment, dans les années 1970 et 1980 au Royaume-Uni, en Allemagne et en Italie. Qu'elles aient pris la forme de guerres sales, de suppression de groupes d'opposition sous des dictatures ou de mesures antiterroristes, il existe une histoire européenne des tensions entre le droit et la violence d'Etat qui a marqué les populations impliquées. L'utilisation de la notion d'état d'exception pour expliquer la suppression de l'Etat de droit dans certaines zones de discrétion (géographiques ou juridiques) s'est accompagnée de l'idée que certaines périodes d'exceptions peuvent se prolonger, devenir routinières, voire normales. Elles ont été incorporées à l'Etat de droit, doucement mais sûrement, non seulement à l'intérieur même de ce que nous considérons comme les limites du normal, mais aussi au sein de la conscience professionnelle du judiciaire. En effet, l'importance de l'Etat de droit, eu égard aux actions de l'administration, a été en partie amoindrie par la multiplication des sources et des portées des dérogations disponibles pour une utilisation dans la vie quotidienne. Il y a ainsi une exception applicable au statut légal des migrants irréguliers, une exception qui façonne en pratique le traitement de jeunes d'origines ethniques différentes et résidant dans des quartiers pauvres tandis que la détention d'étrangers dans des zones aéroportuaires ou dans des camps de transit a ouvert la voie à l'acceptation de la détention illimitée à la prison de Belmarch ou dans les bases américaines de Guantanamo.

8.3. Le climat de malaise et de peur envers certains étrangers a forgé la croyance selon laquelle il existerait un lien entre les pratiques de l'islam et certaines allégeances douteuses à l'Etat. Même lorsque les individus sont devenus des citoyens, leur affiliation religieuse les rend suspects, susceptibles de constituer cette "cinquième colonne » potentielle, représentant «l'ennemi de l'intérieur». En pratique, il s'agit souvent de la manière dont un individu est perçu. Si un enfant est avant tout un étranger, avant même d'être un enfant, le principe contenu dans les législations des droits de l'Homme nationales, européennes, et internationales selon lesquelles la priorité doit être donnée au meilleur intérêt possible de l'enfant dans la manière dont il ou elle est traité(e), passe en second. L'expulsion de l'étranger (même s'il s'agit d'un enfant) a un statut prioritaire sur la protection de l'enfant (bien qu'étranger). La possibilité d'un marquage électronique d'une jeune personne accusée de troubles à l'ordre public et celle de généraliser les procédures de contrôle a également lié ces deux 
dimensions du normal et de l'exceptionnel de manière paradoxale. D'un côté, l'exception envahit le normal et change ce qu'on considère comme normal ; de l'autre, on créé une nouvelle tolérance pour d'autres exceptions. Un climat de peur, voire un état d'urgence permanent a été créé, de sorte que le comportement des individus est façonné par la conviction que nous vivons une période de guerre. Ainsi, qui peut ignorer aujourd'hui à Londres ou à Paris un sac abandonné dans un train ou un bus?

8.4. La conséquence en a été le renforcement de ce que Didier Bigo a appelé en 1998 la "gouvernementalité de la peur» ou le "ban-opticon», dans lesquels il y a une catégorisation de certaines personnes, les rendant sujettes à une exception souveraine, à un exclusion et un bannissement fondés sur la menace représentée par ces personnes. Il existe, pour elles, une normalisation, un impératif dans la limitation de certaines formes de libertés, et, surtout, de la liberté de circulation. Leurs frontières sont délimitées par la sécurisation de la vie de tous les jours, la hausse de l'intolérance et même l'appellation "zéro tolérance ». Le 11 septembre a exacerbé le ban-opticon en rendant plus visibles de nombreuses technologies et rationalités à l'œuvre. Il y a eu, en particulier, une accélération du passage d'un contrôle des personnes sur le territoire, par des moyens permettant leur identification territoriale à un contrôle, à distance via les traces qu'ils laissent. Mais les individus sont également surveillés par rapport à de potentielles actions futures, à partir de ce que l'on sait sur leur passé. Cela explique pourquoi les technologies biométriques sont devenues si essentielles pour la sécurité.

8.5. La réponse aux défis lancés à l'Etat de droit a été jusqu'à présent mitigée. Il y a eu de solides ripostes contre certaines mesures - comme celle de la House of Lords britannique face à la détention illimitée d'étrangers, une législation qu'elle a considérée contraire à la Convention européenne des droits de l'Homme, ou comme le Conseil d'Etat français qui a jugé que la détention des Français à Guantanamo était contraire au droit international - mais un certain nombre de sujets cruciaux sont restés sous silence. La fonction de protection de l'individu que l'Etat de droit devrait occuper n'a pas encore trouvé sa place si l'on considère les quantités importantes de données sur les individus qui sont collectées et utilisées. Pour ces domaines de collecte et d'utilisation de données biométriques, les administrations revendiquent une importante marge de manœuvre, sans que le judiciaire n'ait son mot à dire.

8.6. Le développement progressif d'une « zone de liberté, de sécurité et de justice » en a été particulièrement affecté. Alors que la sécurité est clairement devenue une valeur prédominante, la « liberté » a été reléguée à un rôle secondaire. Depuis que le traité d'Amsterdam, en 1999, a attribué aux polices en charge des questions de liberté, de sécurité et de justice une compétence européenne, il y a eu, dans les discours et dans les politiques mis en place, une tendance à renforcer et à unifier la coopération transnationale pour la sécurité. La sécurité, prise dans le sens d'une protection de l'ordre interne, est devenue l'une des composantes les plus importantes de la citoyenneté européenne dans les domaine de «liberté, sécurité et justice». Cette sphère politique a été fondée sur une méthode à la fois communautaire et intergouvernementale. Elle amène progressivement vers de nouvelles sources de pouvoir discrétionnaire par l'émergence de réseaux de contrôle à l'échelle européenne. En effet, en rendant possible des paramètres normatifs communs et une coordination opérationnelle dans la lutte contre le crime et le terrorisme, la gouvernance de l'Union européenne travaille à renforcer les politiques nationales contre les menaces sécuritaires auxquelles les Etats doivent faire face. 
82 8.7. La déclaration sur la lutte contre le terrorisme du 25 mars 2004 a marqué un nouveau pas vers une approche commune en faveur d'un lot contentieux de propositions restrictives qui ont pour but de lutter contre tout ce qui est perçu comme "activité terroriste». De la même manière, le développement d'un Système d'information Visa (SIV), la seconde génération du Système d'information Schengen (SIS II), ainsi que l'utilisation de la biométrie et de nouvelles technologies de surveillance dans les documents d'identification - visas, permis de séjour, passeports, etc. - sont toujours prioritaires dans la politique de l'Union européenne. La cohérence structurelle et l'effectivité potentielle de toutes ces politiques pro-sécurité, leur compatibilité avec les standards européens et internationaux des droits de l'Homme, la législation sur la protection des données et l'Etat de droit pour les citoyens européens et non européens sont sujets à de sérieux questionnements. Il y a un manque de transparence évident et inquiétant dans l'adoption de ces mesures, dans leur portée et limites véritables, et dans leur relation au " principe de disponibilité » permettant le partage d'informations entre les agences de sécurité dans toute l'Union européenne.

9. La métaphore de l'équilibre et la politique de l'exception

84 9.0. Les chercheurs ELISE sont persuadés que l'application des mesures de sécurité a été bien trop zélée par rapport à la mobilité sociale, à l'immigration et aux politiques d'asile, ainsi qu'à tous les champs sociaux dans lesquels le statut de la liberté est en jeu. Cela ne vise en aucun cas à révoquer les demandes liées aux dangers liés au recours à la violence dans le monde contemporain, mais à souligner que bon nombre de ces revendications ont été produites à partir de récits sur-stimulés, et institutionnellement égocentrés sur ce que signifie le fait de décréter l'état d'urgence, qui garantit la dérogation des libertés, des droits et des responsabilités démocratiques.

85 9.1. Ce jugement n'est peut être pas surprenant d'un point de vue historique ou comparatif. La capacité des agents de la politique à invoquer des dangers imminents pour justifier les restrictions sur les vies des personnes est peut-être l'un des thèmes les plus anciens de l'analyse politique. Il a occupé une place centrale dans les récits sur les origines de l'Etat moderne dans une sorte de contrat social, et il a été essentiel dans la façon dont les Etats modernes ont compris la relation entre la paix chez soi et la guerre chez les autres. Certains iraient même jusqu'à dire que ce qui s'est passé est plus ou moins naturel et tout à fait prévisible. Ceci a également aidé de nombreuses personnes à admettre l'idée selon laquelle il est tout à fait acceptable de privilégier les nécessités de la sécurité par rapport à de nombreuses garanties de libertés.

9.2. Cette apparence de nécessité, d'inévitabilité et de naturalité n'est précisément qu'une apparence. De nombreuses revendications ont été faites à propos des terribles menaces engendrées par les activités terroristes et de la nécessité de mesures exceptionnelles pour y faire face. Mais ces revendications ont été faites par des agences et des groupes professionnels spécifiques, qui ont leurs propres intérêts, rivalités, et myopies. Ils ont été capables de faire valoir leurs connaissances, sans intervention critique, et de créer un environnement dans lequel les connaissances sur les questions de sécurité sont à la fois distinctes des connaissances sur la liberté et bien plus importantes que celles-ci, que l'intégration sociale, que les droits ou que l'Etat de droit. La sécurité est un sujet important. Les actes de terreur ne sont pas insignifiants. Ceci dit, le fait de croire précisément savoir ce qui est important, et dans quelle mesure cela suffit à justifier des formes d'exceptionnalisme devenues largement répandues ces dernières années n'est pas quelque chose que l'on peut ôter du champ de la 
responsabilité démocratique sans que cela n'entraîne de sérieuses conséquences par rapport à ce que nous sommes en droit attendre des sociétés démocratiques.

9.3. Le recours à la métaphore de l'équilibre entre liberté et sécurité est l'une des principales caractéristiques des débats contemporains entre des revendications de sécurité et des revendications de liberté. Cette métaphore "réconfortante " suggère que n'importe qui peut se trouver en position de pouvoir juger, à partir du moment où un équilibre convenable est atteint. Cette métaphore empêche donc de comprendre comment la relation entre ces notions concurrentes est structurellement inégale. Certaines voix ont une légitimité plus grande que d'autres pour se faire entendre, tout comme certains équilibres sont bien plus impressionnants que d'autres. L'équilibre, dans ce cas, suggère une situation de prépondérance, pas d'égalité. Cette métaphore fait courir le risque de penser que tout jugement sur le moment précis où cet équilibre est atteint, est du ressort des agents étroitement liés aux agences de sécurité, même si, finalement, c'est ce dont il s'agit dans l'idée de monopole de la violence détenu par l'Etat souverain. Au contraire, cela encourage les individus à envisager la politique comme une affaire de choix simples, comme si l'on pouvait choisir entre liberté et sécurité, au lieu de les considérer comme des valeurs inséparables sans cesse en conflit potentiel. L'utilisation de l'image «confortable» de la balance dans ce contexte œuvre précisément à dévier l'attention de ce qui est en jeu dans l'idée de l'exception, dans les revendications, afin qu'une décision souveraine soit prise pour suspendre les normes établies et les libertés, au nom de la sécurité dans un état d'exception. Finalement, cette métaphore dévie toutes les questions essentielles sur la responsabilité, le jugement, le traitement des suspects comme des humains ou pas, sur qui en vient à décider qu'un acte de violence garantit une action militaire ou juridique : les dérogations de l'état de droit ou les responsabilités sous l'état de droit.

9.4. Cette métaphore doit, dans la situation actuelle des choses, rendre sceptique, du point de vue du degré de formatage des discours sur la sécurité, comme objets de coopération et d'unification. Les discours sur la liberté sont, eux, formatés en relation à des juridictions fragmentées sévèrement contraintes par le droit et les régulations. La sécurité est désormais considérée comme une valeur sans frontière. La coopération est considérée comme la clef d'une information efficace et centralisée, pour anticiper les événements et déterminer qui est dangereux et qui ne l'est pas, qui est suspect et qui ne l'est pas. En cela, l'Espagne a très largement frayé le chemin aux accords de l'Union européenne, en particulier, pour former une identité européenne, une démocratie unique luttant contre les mêmes ennemis terroristes. Les Etats-Unis souhaiteraient encore plus de coopération au niveau transatlantique, mais sous sa propre direction stratégique. Les sécurités intérieure et extérieure fusionnent par des moyens complexes qui ne cadrent pas avec les récits conventionnels d'un "dilemme sécuritaire » entre Etats, mais qui ont été examinés en détail par les équipes françaises, belges et grecques d'ELISE. D'un autre point de vue, les espaces de la liberté ont été clairement distingués, du point de vue de la liberté de mouvement et de la traversée des frontières, notamment.

9.5. Le défi auquel nous faisons face ne revient pas à identifier un équilibre acceptable entre les appels à la sécurité et les appels à la liberté, mais à effectuer un examen bien plus rigoureux des conditions sous lesquelles les appels à la sécurité garantissent la suspension des libertés. Cela requiert une attention particulière sur les moyens par lesquels la restructuration de la vie politique, en réponse aux nombreuses différentes 
forces, est particulièrement formée et déformée par des agences capables de convertir des menaces sérieuses - exigeant des réponses démocratiques - en états d'urgence extrêmes - exigeant des réponses militaires, de nouvelles modalités de contrôle social, des formes intensifiées de surveillance et d'exclusions et des viols des valeurs fondamentales du libéralisme, de la démocratie et de l'état de droit. de la capacité des agences chargées de garantir une plus grande protection contre les nouvelles formes d'actions discrétionnaires. Ledit « déficit démocratique » chronique dans une grande partie de l'Union européenne est un problème particulièrement sérieux dans ce contexte. Il est donc possible d'envisager une agence pour les droits fondamentaux qui soit indépendante, et capable de concentrer un pouvoir d'inspection administratif sur les agences de sécurité; ou bien un processus d'élargissement d'unification des différentes agences concernées par la protection des données; ou bien des missions spécifiques d'inspection de corps et de groupes européens transnationaux, y compris une surveillance particulière des activités des services de renseignement et de la police, en particulier lorsqu'elles sont opérées par des agents au-delà de leurs frontières étatiques; ou de nouveaux pouvoirs pour permettre des enquêtes sur le comportement de certaines agences de sécurité, qu'elles soient publiques ou privées, avec l'aide, éventuellement, des réseaux d'ONG et d'universitaires de disciplines différentes; ou de nouvelles obligations de responsabilité dans leur utilisation des données pour les Etats membres et les tiers parties qui partagent des données avec l'Union européenne, et qu'ils soient responsables devant la CEDH. D'autres propositions de ce type sont soulignées dans de nombreux textes produits par des groupes de recherche ELISE, en particulier ceux qui impliquent les équipes grecques et belges, mais aussi celles qui se sont intéressées aux nouvelles procédures en matière d'immigration, de citoyenneté, de maintien de l'ordre et de droit.

10.2. Toutefois, d'une manière ou d'une autre, de telles initiatives institutionnelles doivent être accompagnées par un engagement renouvelé pour une politique de la responsabilité : par une volonté de résister aux perceptions séduisantes sur la nécessité de bafouer les libertés au nom de la sécurité, et de garantir que les exceptions aux attentes normales de liberté, d'égalité et de démocratie dans le cadre de l'Etat de droit ne soient faites que dans le cadre d'une évaluation soutenue et pluridimensionnelle. Il n'y a aucune raison d'abandonner les résultats de la vie politique moderne à ceux à qui l'on a permis de parler au nom de la sécurité et uniquement de la sécurité. Mais il y a de fortes raisons de croire qu'on a laissé beaucoup trop de terrain aux agences et aux institutions qui ont pris l'habitude de parler de cette manière. Bien que les menaces de 
terreur et de violence puissent se répéter, il ne faut pas omettre la possibilité d'excès d'une nouvelle politique de l'exception. Il ne s'agit pas simplement des «libertés civiles ", mais des principes fondamentaux de la vie politique moderne. Finalement, ce sujet ne peut être laissé aux mains des professionnels de la sécurité, ni même à celles des professionnels de la politique. Si l'on considère la crise générée par la réponse publique négative au projet de Constitution européenne, le temps est venu de mettre en place des discussions sur une base bien plus large.

\section{NOTES}

1.. Traduction collective par l'équipe de Cultures \& Conflits.

\section{RÉSUMÉS}

La liberté constitue toujours le principe à partir duquel toutes les interférences des Etats en termes de sécurité doivent être limitées, justifiées et transparentes dans les traités de l'Union européenne. La perspective qui s'esquisse ici, avec les projets de recherches plus spécifiques qui en font partie, suggère que la résistance face à la marginalisation des revendications de liberté doit être plus forte dès lors que l'on invoque le besoin d'une sécurité accrue. En d'autres termes, là où les possibilités de liberté politique sont désormais retenues par des formes de fragmentation structurelle et institutionnelle, elles devraient se nourrir des formes imaginaires de coopération entre les juridictions existantes; et là où les possibilités de coopération et d'unification sont pensées pour contrôler les populations dans une plus grande ampleur, elles devraient faire l'objet d'un contrôle démocratique plus important, qu'il soit parlementaire ou qu'il vienne de communautés et d'institutions démocratiques responsables et transparentes. Les implications en termes de politiques, que nous mettons en avant dans ce texte, suivent les principes que nous venons d'exposer.

In EU Treaties «liberty» is always the principle against which any state interference on the basis of security must be limited, justified and open to judicial scrutiny. The perspective sketched here, along with the more specific research projects that inform it, suggest an urgent need for much more robust resistance to the marginalization of claims about liberty whenever the necessities of security are invoked. In general terms it might be said that where the possibilities of political liberty are currently being constrained by forms of structural and institutional fragmentation, they ought to be nurtured by imaginative forms of cooperation across existing jurisdictions; and where the possibilities of cooperation and unification are being sought in order to control human populations on a wider scale, they ought to be subject to greater scrutiny and control by many different democratically accountable communities and institutions. The policy implications advanced in this paper follow these principles. 
INDEX

Index géographique : Europe

Mots-clés : construction européenne, libertés publiques, sécurité 